\title{
A novel colonoscope with panoramic visualization detected more simulated polyps than conventional colonoscopy in a live swine model
}

Authors

Institutions
Nathan Gluck ${ }^{1,2}$, Sigal Fishman ${ }^{1,2}$, Alaa Melhem ${ }^{1,2}$, Sharon Goldfarb ${ }^{3}$, Zamir Halpern ${ }^{1,2}$, Erwin Santo ${ }^{1,2}$

${ }^{1}$ Tel Aviv Medical Center, Tel-Aviv, Israel.-

${ }^{2}$ Sackler Faculty of Medicine, Tel-Aviv University, Tel-Aviv, Israel

${ }^{3}$ GI View Ltd, Ramat Gan, Israel.

\section{Bibliography}

DOI http://dx.doi.org/

$10.1055 / \mathrm{s}-0034-1393080$

Published online: 6.10.2015

Endoscopy International Open 2015; 03: E642-E645

(c) Georg Thieme Verlag KG

Stuttgart · New York

E-ISSN 2196-9736

\section{Corresponding author:}

Nathan Gluck, MD, PhD

Tel-Aviv Sourasky Medical Center

Research Center for Digestive Disorders and Liver Diseases

6 Weizmann St

Tel-Aviv 64239

Israel

Phone: 9723-6974282

Fax: 9723-6974622

nathang@tlvmc.gov.il

\section{License terms}

(1) $\Theta \circledast$
Background and study aims: The Aer-O-Scope ${ }^{\mathrm{TM}}$ Colonoscope System (AOS) combines panoramic $360^{\circ}$ view with standard forward view. We assessed the AOS's ability to identify lesions implanted in live swine, compared to conventional colonoscopy (CC).

Patients and methods: Twelve swine colons were surgically ligated and beads sewn within. Five procedures ( 3 AOS and $2 \mathrm{CC}$ ) were performed on each swine and findings reported. Physicians were blinded to number, size, and color of beads. The sequence of procedures and physicians was randomized. Pigs, physicians, and colonoscopes were randomly alternated between examination rooms, maintaining physician blindness. Two in-

\section{Introduction \\ $\nabla$}

Missed adenomas during screening colonoscopy remain a major concern and a leading cause of interval cancer [1], making the adenoma detection rate (ADR) an important indicator of quality performance for the endoscopist [2]. Polyps that arise in concealed locations can be difficult to detect with $140^{\circ}$ to $170^{\circ}$ forward-viewing conventional colonoscopy (CC) and may account for a proportion of missed adenomas [3]. A number of colonoscopic devices that improve detection of these polyps have recently been introduced $[4,5]$.

The Aer-O-Scope ${ }^{\mathrm{TM}}$ Colonoscope System (GI View Ltd., Ramat-Gan, Israel) (AOS) is a disposable gaspropelled colonoscope with a visualization system that utilizes a novel optical concept designed to match the colon anatomy: a circumferential omni-directional $360^{\circ}$ panoramic viewer (OMNI view) projects a $360^{\circ}$ view of the lumen [6]. An additional conventional forward view ( $57^{\circ}$ field of view) is provided. The recent addition of joystick-controlled steering for the colonoscope allows for simpler navigation and centering control during withdrawal scan. Pathologies are visualized in both views which are complementary, re- dependent blinded physicians interpreted procedure videos offline.

Results: A total of $259 / 273$ (94.9\%) of lesions were visualized by AOS compared to $158 / 182$ with CC $(86.8 \%)(P=0.002)$. Miss rates of lesions $\geq 6 \mathrm{~mm}$ were $2.6 \%$ and $10.5 \%$, respectively $(P=$ 0.022 ), and $6.9 \%$ and $15.1 \%$, respectively, for lesions $<6 \mathrm{~mm} \quad(P=0.031)$. Mean agreement between AOS and CC for lesion detection was $88.3 \%$. The benefit of AOS was maintained in offline video review.

Conclusions: AOS, featuring panoramic $360^{\circ}$ view, demonstrated high detection rates for simulated colonic lesions in a live swine model.

sulting in a substantially larger visualized area of the colonic mucosa. These combined views are projected onto a single, user-friendly screen with the OMNI view surrounding the forward view $(\bullet$ Fig. 1 a and $\bullet$ Fig. 1 b)

The visualization properties of the AOS have been enhanced recently: New LEDs with improved orientation provide greater coverage and higher light uniformity, gamma control improves visibility in darker areas of the colon, a new front lens eliminates front-view distortion, and full digital video pass from the optical head to the screen removes analogue noise.

In this comparison to forward-viewing $\mathrm{CC}$, we aimed to evaluate the performance of the AOS in identifying simulated polyp-like lesions in an in vivo swine model, prior to its introduction for clinical studies.

\section{Patients and methods}

$\nabla$

\section{Swine model}

This was a prospective live swine model study performed at the Lahav Animal Research Institute, Lahav, Israel. Twelve female swine (Sus scro- 

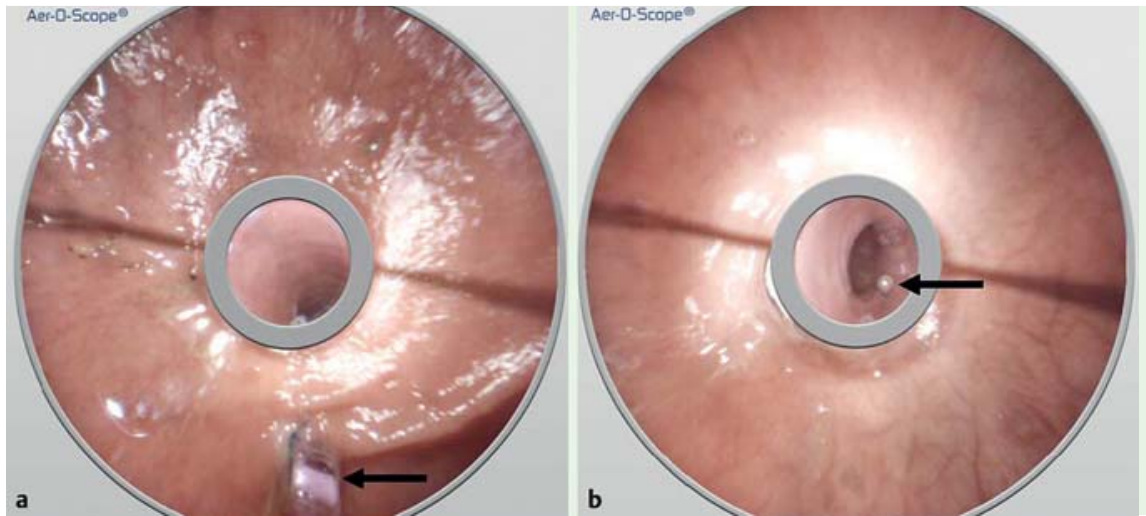

Fig. 1 Beads visualized by Aer-O-Scope ${ }^{\mathrm{TM}}$ Colonoscope. a Bead in panoramic (OMNI) view. b Bead in forward facing view. fa domestica-large white), aged 3.5 to 4.5 months and weighing 65 to $80 \mathrm{~kg}$ were studied. Animal care, facilities, and activities were approved and monitored according to IS09001 standards for quality and service. The Tel Aviv University Animal Ethics Committee approved the study. Bowels were cleansed using Soffodex and fleet enemas. Subsequently, simulated colon polyps were surgically introduced under general anesthesia. Because the swine colon is tortuous, the colon was ligated with thread just beyond the first sharp colonic curve $(100-120 \mathrm{~cm}$ from the anus) to facilitate colonoscope passage. Next, six to 10 colored beads measuring 2.5 to $10 \mathrm{~mm}$ in diameter were then introduced per animal in a preset random order distal to the ligation (total of 91 beads placed in 12 swine). To better simulate polyps, half the beads (46/91) were pushed from outside the bowel wall inward and tied at their base, resulting in mucosal coating of the bead ("pseudopolyps") ( Fig.2). All other beads were surgically sutured to the colonic mucosa through a small incision in the colonic wall. The endoscopists were blinded to all implanted beads.

\section{Endoscopist AOS training}

Two gastroenterologists (NG \& SF) highly experienced in colonoscopy ( $>5$ years) attended a 1-hour lecture and performed three AOS procedures on swine with implanted beads ( 3 hours).

\section{Study protocol}

Each of the 12 swine underwent a total of three AOS and two CC (Olympus CF 140 L SD) procedures (study total 60; 36 AOS and 24 $\mathrm{CC}$ ). Lesions were counted and recorded during withdrawal phase.

Several measures were taken to ensure that endoscopists were blinded to which pig was being examined at any given time, thus preventing them from recalling previously viewed pathologies within the colon of a specific animal. Procedures were ran-

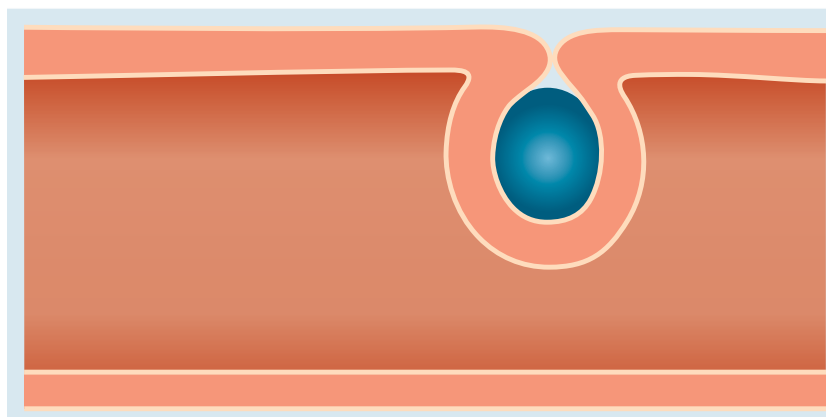

Fig. 2 Schematic of simulated polyp implantation in wall of swine colon. domly assigned to the two endoscopists, procedures were performed in two separate rooms on two different swine simultaneously, and the sequence of procedures (AOS or CC) in each swine was random. The one exception to the random sequence was the condition that six swine would begin the study with an AOS while six would begin with CC. This exception was made to prevent bias and to neutralize the "second pass" effect of increased polyp detection in tandem colonoscopy [7]. Swine, physicians, and colonoscope towers also were randomly alternated between procedure rooms. To facilitate that, physicians had to leave rooms post procedures.

In order to neutralize polyp-miss due to momentary lack of attention or quick motion through curves, all procedures were video recorded and later reviewed by two additional highly experienced endoscopists (ES \& AM), who were blinded to swine, pathologies and the performing endoscopist. These physicians could stop the video, go back and review sections at their discretion.

To determine the effectiveness of the randomization scheme in preventing recall, miss rates for successive procedures (first, second, and third passes) for AOS and CC, online and offline, were compared.

\section{Study endpoints}

The study was designed to demonstrate non-inferiority of the AOS relative to CC: the number of simulated polyps detected by AOS in a given swine was at least the same as with CC in two out of three repeated procedures. Alternatively, the number detected in at least six animals would be as good as or better than CC.

\section{Sample size calculation and statistical analysis}

Sample size was based on the assumption that the equivalence ratio of AOS to CC (non-inferiority) by pre-defined criteria was $\geq 80 \%$. A sample of 12 animals would then provide power $\geq 80 \%$ (type II error $<20 \%$, type I error $<5 \%$ ). This included an estimated dropout rate (such as swine death unrelated to the procedure) of $10 \%$.

Tests for calculating simulated polyp detection rates were 2-sided using Pearson Chi-square. The threshold for a significant $P$ value was 0.05 .

\section{Results}

- Table 1 lists detection and miss rates for Aer-O-Scope colonscopy vs. conventional colonoscopy as reported during colonoscopy. Overall, significantly more implanted beads were detected using 
Table 1 Detection and miss rates for Aer-O-Scope colonoscopy vs. conventional colonoscopy: live endoscopy procedure data.

\begin{tabular}{|c|c|c|c|c|c|}
\hline & Colonoscope type & $\begin{array}{l}\text { Total beads implanted } \\
\text { in all Procedures }\end{array}$ & $\begin{array}{l}\text { Percent of beads visualized } \\
\text { (bead no.) }\end{array}$ & $\begin{array}{l}\text { Miss rate } \\
\text { (\%) }\end{array}$ & $\begin{array}{l}\text { Pearson } X^{2} \\
P \text { value }\end{array}$ \\
\hline \multirow[t]{2}{*}{ Overall implanted beads } & Aer-O-Scope & 273 & $94.9(259)$ & 5.1 & 0.002 \\
\hline & Conventional & 182 & $86.8(158)$ & 13.2 & \\
\hline \multirow[t]{2}{*}{ Beads $\geq 6$ mm } & Aer-O-Scope & 114 & $97.4(111)$ & 2.6 & 0.022 \\
\hline & Conventional & 76 & $89.5(68)$ & 10.5 & \\
\hline \multirow[t]{2}{*}{ Beads $<6 \mathrm{~mm}$} & Aer-O-Scope & 159 & $93.1(148)$ & 6.9 & 0.031 \\
\hline & Conventional & 106 & $84.9(90)$ & 15.1 & \\
\hline
\end{tabular}

\begin{tabular}{|c|c|c|c|c|c|}
\hline & Colonoscope type & $\begin{array}{l}\text { Total beads implanted } \\
\text { in all Procedures }\end{array}$ & $\begin{array}{l}\text { Percent of beads visualized } \\
\text { (bead no.) }\end{array}$ & $\begin{array}{l}\text { Miss rate } \\
\text { (\%) }\end{array}$ & $\begin{array}{l}\text { Pearson } X^{2} \\
P \text { value }\end{array}$ \\
\hline \multirow[t]{2}{*}{ Overall implanted beads } & Aer-O-Scope & 273 & $95.6(261)$ & 4.4 & 0.034 \\
\hline & Conventional & 182 & $90.7(165)$ & 9.3 & \\
\hline \multirow[t]{2}{*}{ Beads $\geq 6 \mathrm{~mm}$} & Aer-O-Scope & 114 & $98.2(112)$ & 1.8 & 0.365 \\
\hline & Conventional & 76 & $96.1(73)$ & 3.9 & \\
\hline \multirow[t]{2}{*}{ Beads $<6 \mathrm{~mm}$} & Aer-O-Scope & 159 & $93.7(149)$ & 6.3 & 0.055 \\
\hline & Conventional & 106 & $86.8(92)$ & 13.2 & \\
\hline
\end{tabular}

Table 3 Detection of pseudopolyps and exposed beads.

\begin{tabular}{|llllllll|} 
& $\begin{array}{l}\text { AOS live total } \\
\text { visualized }\end{array}$ & $\begin{array}{l}\text { CC live total vis- } \\
\text { ualized }\end{array}$ & $\begin{array}{l}\text { AOS review } \\
\text { total visualized }\end{array}$ & $\begin{array}{l}\text { CC review total } \\
\text { visualized }\end{array}$ & $\begin{array}{l}\text { Total \% missed } \\
\text { (live) }\end{array}$ & $\begin{array}{l}\text { Total \% missed } \\
\text { (review) }\end{array}$ & $\begin{array}{l}\text { Total \% missed } \\
\text { (overall) }\end{array}$ \\
\hline Pseudopolyps & $129 / 135$ & $78 / 90$ & $131 / 135$ & $82 / 90$ & 8.0 & 5.3 & 6.7 \\
\hline Beads & $130 / 138$ & $80 / 92$ & $130 / 138$ & $83 / 92$ & 8.7 & 7.4 & 8.0 \\
\hline
\end{tabular}

AOS, Aer-O-Scope Colonoscope System; CC, conventional colonoscopy

\begin{tabular}{lllll}
\hline $\begin{array}{l}\text { Table } 4 \\
\text { Pass \# }\end{array}$ & $\begin{array}{c}\text { Detection rates by successive pass } \\
\text { AOS detected/total lesions (\%) }\end{array}$ & $\begin{array}{l}\text { AOS offline review detected/ } \\
\text { total lesions (\%) }\end{array}$ & CC detected/total lesions (\%) & $\begin{array}{l}\text { CC offline review detected/ } \\
\text { total lesions (\%) }\end{array}$ \\
\hline 1 & $86 / 91(95)$ & $87 / 91(96)$ & $80 / 91(88)$ & $87 / 91(96)$ \\
\hline 2 & $85 / 91(93)$ & $87 / 91(96)$ & $78 / 91(86)$ & $79 / 91(87)$ \\
\hline 3 & $88 / 91(97)$ & $88 / 91(97)$ & - & - \\
\hline
\end{tabular}

AOS, Aer-O-Scope Colonoscope System; CC, conventional colonoscopy

AOS [259/273 (94.9\%)] as compared to CC [158/182 (86.8\%)] (P $=0.002)$. Interestingly, 20/259 beads detected by AOS $(7.3 \%$ of 273 implanted beads) were visualized exclusively by the forward viewing mode, indicating a complementary role for this view. The mean agreement rate of AOS with CC for lesion detection or miss was $88.3 \%$. Subgroup analysis, stratified by polyp size 1 to $5 \mathrm{~mm}$ or $\geq 6 \mathrm{~mm}$, also demonstrated a statistically significant advantage for AOS in both subgroups.

During post-endoscopy video review, significantly more implanted beads were detected by AOS [261/273 (95.6\%)] as compared to CC [165/182 (90.8\%)], $(P=0.034)(\checkmark$ Table 2$)$. The mean agreement rate of AOS with CC for lesion detection or miss was $92.1 \%$. Subgroup analysis stratified by size of polyps showed a trend toward an advantage for AOS, though without statistical significance ( $P$ value $=0.055$ for beads $<6 \mathrm{~mm}$ and 0.365 for beads $\geq 6 \mathrm{~mm}$ ). There were no statistical differences between visualization of exposed beads compared to "pseudopolyp"-like beads by either AOS or CC, neither during live procedures nor offline video review (- Table 3).

We wished to ascertain whether the increased number of AOS procedures performed relative to $\mathrm{CC}$ could bias toward better detection, and whether the randomization scheme was effective in preventing physicians' recollection of consecutive colonoscopies on the same swine. As seen in Table4, the difference between AOS and CC was already evident in the first passes, and the detection rates did not improve after several successive procedures as might have been expected.

Complete intubation to the depth of colon ligation was achieved in $60 / 60(100 \%)$ procedures. No adverse events were experienced in any of the procedures. These were not study endpoints. 


\section{Discussion}

\section{$\nabla$}

This comparative study demonstrates the efficacy of AOS, a novel disposable colonoscope, for simulated polyp detection in a swine model. The advantage of the AOS over CC was demonstrated overall as well as in lesions of clinically significant size $(\geq 6 \mathrm{~mm})$. Notably, training on the AOS was completed in just half a day, despite differences in manual technique and image display (৫ Fig. 1).

Previous AOS studies have shown images of native swine colon or of ex-vivo sewn beads obtained with forward and OMNI panoramic views, and safety of use in live swine $[6,8]$. The current study is the first statistically significant, in-vivo comparative blinded study to test the simulated polyp detection ability of the AOS.

The study design, in which the number of pathologies was given, enabled us to accurately compare the absolute miss rates of both the AOS and CC. Miss rates in both modalities were inversely related to polyp size. Interestingly, CC miss rates in this study were lower than in human tandem colonoscopy studies [9], supporting the adequacy of performance by the operators. Another strength of the study is the use of four independent physicians. Some polyps were missed by performing endoscopists in both AOS and CC (more so in the CC group), despite being visible on video recordings. Despite not being part of real-world practice, offline video review adds objectivity to the comparison of the devices and is becoming an accepted quality indicator tool for polyp detection studies [10], prompting us to include these data. An advantage for AOS was evident both in post-procedural reports (when findings are typically reported in clinical practice) and in later video analysis. The offline review also allowed for a larger sample size and physician pool for statistical analysis.

There are several limitations to this study. Porcine anatomy differs from the human colon in having no haustral folds. Thus, the reason for improved detection by AOS relative to CC becomes less clear. In addition, the colonoscope used in this study, although providing high-quality images, has somewhat less field of view $\left(140^{\circ}\right)$ than currently available technology $\left(170^{\circ}\right)$. Procedures were repeated in 12 swine, potentially resulting in physicians remembering specific, previously scoped swine colons. However, this appears to have been ameliorated by the randomization scheme ( $\bullet$ Table 4$)$.
In conclusion, the Aer-O-Scope gas-propelled colonoscope, featuring a novel $360^{\circ} \mathrm{OMNI}-$ directional view, provided excellent simulated polyp detection rates in this in vivo swine study and may show an advantage over CC. The rapid training of the physicians operating this system did not appear to compromise the study results.

Further studies of screening colonoscopy in humans are warranted to validate the clinical relevance of these animal model findings.

Competing interests: Dr. Halpern has received consulting fees from GI View Ltd. Ms. Goldfarb is currently an employee at GI View Ltd.

\section{References:}

1 Leufkens AM, van Oijen MG, Vleggaar FP et al. Factors influencing the miss rate of polyps in a back-to-back colonoscopy study. Endoscopy 2012; 44: 470-475

2 Kaminski MF, Regula J, Kraszewska E et al. Quality indicators for colonoscopy and the risk of interval cancer. N Engl J Med 2010; 362: $1795-1803$

3 Jover R, Zapater P, Polania E et al. Modifiable endoscopic factors that influence the adenoma detection rate in colorectal cancer screening colonoscopies. Gastrointest Endosc 2013; 77: 381 - 389 e1

4 Gralnek IM, Segol O, Suissa A et al. A prospective cohort study evaluating a novel colonoscopy platform featuring full-spectrum endoscopy. Endoscopy 2013; 45: 697-702

5 Leufkens AM, DeMarco DC, Rastogi A et al. Effect of a retrograde-viewing device on adenoma detection rate during colonoscopy: the TERRACE study. Gastrointest Endosc 2011; 73: 480-489

6 Arber N, Grinshpon R, Pfeffer J et al. Proof-of-concept study of the Aer$\mathrm{O}$-Scope omnidirectional colonoscopic viewing system in ex vivo and in vivo porcine models. Endoscopy 2007; 39: 412-417

7 Rex DK, Cutler CS, Lemmel GT et al. Colonoscopic miss rates of adenomas determined by back-to-back colonoscopies. Gastroenterology 1997; $112: 24-28$

8 Pfeffer J, Grinshpon R, Rex D et al. The Aer-O-Scope: proof of the concept of a pneumatic, skill-independent, self-propelling, self-navigating colonoscope in a pig model. Endoscopy 2006; 38: 144-148

9 van Rijn JC, Reitsma JB, Stoker J et al. Polyp miss rate determined by tandem colonoscopy: a systematic review. Am J Gastroenterol 2006; 101: $343-350$

10 Rex DK. Looking over your shoulder during colonoscopy: potential roles for videorecording colonoscopy withdrawals. Gastrointest Endosc 2012; 75: $134-137$ 